\title{
Long-term effects of sodium pentobarbital anesthesia on novelty-induced behaviors
}

\author{
ROB McGEE, PETER POWER, and PETER GOLUS \\ The University of Newcastle, Newcastle, N.S.W. 2308, Australia
}

\begin{abstract}
Two experiments were performed to evaluate the long-term effects of a single anesthetic dose of sodium pentobarbital on novelty-induced behaviors. In Experiment 1, when compared with controls 1 week after injection, pentobarbital-treated rats showed faster step-down latencies in a novelty box and an enhanced plasma corticosterone response to this novel environment. In Experiment 2, when exposed to a novel open field, pentobarbital treated animals showed significantly less ambulation and rearing than did control animals. As in the previous experiment, initial latency to move was significantly faster. Previous findings have demonstrated long-term effects of pentobarbital anesthesia on neurochemical and behavioral indices. The present results reemphasize that the consequences of anesthesia may be more prolonged than previously presumed.
\end{abstract}

Studies investigating the behavioral effects of barbiturate administration have typically tested the animals a relatively short time after injection of the drug. In the short-term, barbiturates such as sodium pentobarbital have been shown to affect a variety of behaviors in experimental animals. For example, Cunha and Masur (1978) demonstrated that a $20-\mathrm{mg} / \mathrm{kg}$ dose of sodium pentobarbital in rats had the general effect of decreasing activity in the open field. This was indicated by reduced ambulation and rearing and longer periods of immobility. Low doses of pentobarbital can induce eating and drinking in satiated rats (Jacobs \& Farel, 1971; Watson \& Cox, 1976) and also appear to attenuate taste-neophobia in fluid-deprived animals (Rondeau, Jolicoeur, Kachanoff, Scherzer, \& Wayne, 1975).

Recently, McGee, Golus, and Russell-Jones (1980) showed that a single anesthesia-inducing dose of sodium pentobarbital reduced the taste neophobia exhibited by rats to a novel saccharin solution 1 week after drug administration. This finding confirmed an earlier observation by McGee and Golus (1980) that presurgical pentobarbital anesthesia in female rats attenuated taste neophobia 18 days after the operation. In addition, Power, Myers, Golus, and McGee (1980) found elevated whole-brain serotonin levels in male rats 1 week after a single injection of $60 \mathrm{mg} / \mathrm{kg}$ sodium pentobarbital. While maternal consumption of barbiturates has long-term effects on the behavior of the offspring (Harris \& Case, 1979), the above studies suggest that long-term behavioral and bio-

R. McGee is now at: Department of Paediatrics and Child Health, Medical School, University of Orago, Dunedin, New Zealand. Reprint requests may be sent to P. Golus, Department of Psychology, University of Newcastle, Newcastle, N.S.W. 2308, Australia. chemical changes may occur in adult rats given a single injection of a barbiturate.

It is of interest that the effects of pentobarbital observed 1 week after administration mirror those found in the short term. Our findings of attenuated taste neophobia 7 days after pentobarbital administration supports the results of Rondeau et al. (1975). Kuschinsky, Seidel, Reetz, and Meyer-Burgdorf (1973) found elevated brain serotonin levels in mice 60 and $90 \mathrm{~min}$ after injection of $50 \mathrm{mg} / \mathrm{kg}$ sodium pentobarbital, a finding also noted by Power et al. (1980) 1 week after drug administration. The aim of the present experiments was to investigate the effects of pentobarbital anesthesia on the behavior of rats exposed to a novel environment. In the short term, Cunha and Masur (1978) reported diminished activity in the open field following barbiturate administration. The question of interest is whether such effects are evident after a longer delay.

\section{EXPERIMENT 1}

The first study was designed to examine the behavioral effects of sodium pentobarbital anesthesia 1 week after administration. In addition to activity measures, plasma corticosterone (11-OHCS) levels were also assayed following exposure to the novel environment.

\section{Method}

Subjects. Sixteen male Wistar rats, aged 90-100 days, were used. Upon arrival in the laboratory, they were housed individually in wire-mesh cages and given free access to food and water. They were maintained on a 12:12 h light:dark cycle with lights off at $1200 \mathrm{~h}$.

Apparatus. The novel environment used in testing was a clear Plexiglas box $(20 \times 25 \times 45 \mathrm{~cm})$ mounted on a grid floor consisting of brass rods $.5 \mathrm{~cm}$ thick and set $1 \mathrm{~cm}$ apart. In one corner 
there was a $15 \times 15 \mathrm{~cm}$ platform raised $3.5 \mathrm{~cm}$ above the floor and balanced on a microswitch that could be closed by the weight of the rat. This switch was linked to a logic unit which activated a printout timer and electronic clock. The weight of the rat on the platform started the clock, which stopped when the animal stepped down onto the grid floor.

Procedure. After 2 weeks' adaptation to the laboratory conditions, the rats were assigned to one of two groups $(n=8)$. One group was given a single injection of sodium pentobarbital (Nembutal) at a dose of $60 \mathrm{mg} / \mathrm{kg}$, while the other was given a control injection of an equivalent volume $(1 \mathrm{ml} / \mathrm{kg})$ of physiological saline. Anesthesia was noted in all pentobarbital-treated animals. These injections took place between 900 and $1000 \mathrm{~h}$, prior to lights off. The rats were then left undisturbed for 1 week except for routine cleaning and feeding.

Testing occurred 1 week after the injections, between the 9th and 11 th hours of darkness $(2100-2300 \mathrm{~h})$. This period was chosen because it is at the trough of the circadian rhythm cycle of corticosterone (Pfister \& King, 1976) and thus maximizes the possible rise in plasma 11-OHCS in response to stress. Each animal was removed from the holding room in its home cage and taken to an adjoining room which contained the test apparatus. It was then removed from the home cage and placed on the raised platform in the novelty box, thereby activating the timer. A single observer remained in the room and recorded the frequency of rearing (number of times the animal stood on hindlegs) and defecation (number of boluses) in a 20-min period. At all times, the holding room, test room, and general laboratory area were illuminated by red light. At the end of this time, the rat was removed to the general laboratory area and sacrificed by decapitation to collect blood for 11-OHCS assay. The blood was collected in heparinized tunes and centrifuged, and the plasma was frozen. Plasma 11-OHCS was assayed using the fluorimetric method described by Mattingley (1962).

\section{Results and Discussion}

Table 1 shows the mean scores for the pentobarbital and control animals on step-down latency, total rearing, defecation, and plasma 11-OHCS. In the analysis of the latency scores, an $\mathrm{F}$ test comparing the variances of the two groups was significant $\left(\mathrm{F}_{\max }\right.$ $=6.16, \mathrm{p}<.05$ ), indicating that pentobarbital had reduced the variability of the scores. For this reason, the Mann-Whitney $U$ test, a nonparametric test, was used to compare the latency scores, with $U=13$, $\mathrm{p}<.05$, for a two-tailed test. Step-down latency was faster for those rats receiving the barbiturate.

The rearing, defecation, and 11-OHCS values were analyzed with two-tailed $t$ tests. The two groups did not differ significantly on either rearing $[\mathrm{t}(14)=1.32]$ or defecation $[t(14)=0.47, p>.05]$. There was a sig-

Table 1

Mean Values and Standard Errors for Step-Down Latency

(Seconds), Rearing, Defecation, and Plasma 11-OHCS

$(\mu \mathrm{g} / 100 \mathrm{ml})$ in the Pentobarbital and Control Conditions in Experiment 1

\begin{tabular}{lrr}
\hline & \multicolumn{2}{c}{ Condition } \\
\cline { 2 - 3 } Measure & Pentobarbital & \multicolumn{1}{c}{ Control } \\
\hline Latency & $19.40 \pm 6.50$ & $52.76 \pm 16.13$ \\
Rearing & $38.12 \pm 6.02$ & $47.75 \pm 4.12$ \\
Defecation & $3.00 \pm 0.96$ & $3.75 \pm 1.28$ \\
11-OCHS & $37.95 \pm 1.93$ & $28.35 \pm 2.06$ \\
\hline
\end{tabular}

nificant difference, however, in the case of 11-OHCS, with $\mathrm{t}(14)=3.40, \mathrm{p}<.01$. Plasma 11-OHCS levels were significantly higher in the pentobarbital rats than in the controls.

In summary, pentobarbital administered 1 week prior to testing resulted in shorter step-down latencies and an enhanced 11-OHCS response to a novel environment. It is worth noting that although the difference in total rearing was not significant by a t test, it was significant with a sign test, indicating that this might be worth further investigation. Finally, there was no effect of drug treatment on defecation.

\section{EXPERIMENT 2}

The aim of this experiment was to further examine the long-term effect of pentobarbital on behavioral measures in a novel environment. In this study, the novel environment used was the open field, as in Cunha and Masur (1980). This allows for the measurement of ambulation as an indicator of general activity, as well as the latency, rearing, and defecation measures used in Experiment 1.

\section{Method}

Subjects. Fourteen male Wistar rats aged 90-100 days were used in this study. They were housed under laboratory conditions similar to those in Experiment 1.

Apparatus. The apparatus was based on the open field described by Broadhurst (1960), and consisted of a circular, plywood enclosure, $80 \mathrm{~cm}$ in diameter, with a wall $30 \mathrm{~cm}$ high. The floor was further subdivided by three concentric circles and radiating lines from the center into 19 segments of equal area.

Procedure. After adaptation to the laboratory conditions, rats were divided into two equal groups $(n=7)$, one of which received $60 \mathrm{mg} / \mathrm{kg}$ sodium pentobarbital and the other a control injection of physiological saline. One week later, the animals were tested between 2100 and $2300 \mathrm{~h}$ in the open-field apparatus, under red light conditions. The measures taken included the latency to move from the center circle in the open field, the number of times the rats reared on its hindlegs, ambulation as measured by the total number of floor segments entered, and defecation.

\section{Results}

Table 2 shows the mean values for latency, rearing, ambulation, and defecation in the pentobarbital and control conditions. The results were analyzed with one-tailed $t$ tests based on the direction of the

Table 2

Mean Values and Standard Errors for Latency (Seconds), Rearing, Ambulation, and Defecation in the Pentobarbital and Control Conditions in Experiment 2

\begin{tabular}{lrr}
\hline & \multicolumn{2}{c}{ Condition } \\
\cline { 2 - 3 } Measure & \multicolumn{1}{c}{ Pentobarbital } & \multicolumn{1}{c}{ Control } \\
\hline Latency & $15.32 \pm 2.79$ & $25.00 \pm 4.01$ \\
Rearing & $39.57 \pm 6.89$ & $67.71 \pm 7.09$ \\
Ambulation & $137.43 \pm 19.84$ & $208.57 \pm 25.93$ \\
Defecation & $6.00 \pm 1.38$ & $5.29 \pm 0.97$ \\
\hline
\end{tabular}


findings in the previous study. As in Experiment 1, latencies were slower in the control animals than in the pentobarbital treated rats $[\mathrm{t}(12)=1.98, \mathrm{p}<.05]$. Both rearing $[\mathrm{t}(12)=2.85]$ and ambulation $[\mathrm{t}(12)=$ 2.18 ] were significantly $(p<.05)$ reduced in the pentobarbital group, but there was no difference on the defecation measure $[\mathrm{t}(12)=.42, \mathrm{p}>.05]$.

\section{GENERAL DISCUSSION}

The results of the two experiments suggest that sodium pentobarbital, in a dosage that induces anesthesia, can influence behavior in response to novelty 7 days after administration. As such, the findings confirm those of McGee and Golus (Note 1), McGee et al. (1981), and Power et al. (1980) in suggesting long-term behavioral and biochemical changes following pentobarbital anesthesia. In the present experiments, the changes were revealed as shorter latencies to move upon initial exposure to the novel environment, reduced exploratory activity (rearing and ambulation), and a higher 11-OHCS response. In this regard, Cunha and Masur (1978) have reported reduced locomotor activity immediately following pentobarbital injection also.

The behavior of the animals receiving pentobarbital suggests that the drug heightened the animals' reactivity upon exposure to the novel environments. This heightened reactivity is indicated by the shorter latency (in both experiments) to move upon initial exposure to the environment and also by the higher glucocorticosterone response of the pentobarbital group. It is of note that the anesthetic does not alter baseline 11-OHCS levels (Power et al., 1980). Rather, the effect of the drug is to alter the physiological response in the presence of strong, novel stimulation. Jacobs and Farel (1971) have similarly argued that barbiturates may potentiate arousal in the presence of a strong stimulus.

A second characteristic of the rats administered pentobarbital was their lower level of exploratory activity in the environment. This reduced activity may be indicative of behavioral inhibition in the continued presence of a stressful or threatening situation. One possibility is that the reduction of exploratory behavior is mediated by the elevated serotonin levels found in rats 1 week after pentobarbital administration (Power et al., 1980). Levels of brain serotonin (5-HT) have been implicated in the observed levels of locomotor activity and tonic immobility in various animals (e.g., Hennig, 1979). Both locomotor activity and tonic immobility, in turn, are presumed to be related to fear or threat. At present, however, the mechanism via which pentobarbital produces these behavioral effects is not clear.

The effects of sodium pentobarbital anesthesia on activity in novel environments, taste neophobia, and 11-OHCS and 5-HT levels argue that the consequences of the anesthesia may be more prolonged than normally presumed. As such, anesthesia used for surgical purposes in behavioral research may alter the biochemistry and behaviors of experimental animals over and above any intervention, for example, lesioning produced under anesthesia. What needs to be determined is the duration of these effects. Are they present, for example, 3 or 4 weeks after anesthesia. A second question requiring further investigation is whether all anesthetics have these unwanted side effects. It would be desirable to use an anesthetic for research purposes which produces the least alteration in the experimental animal.

\section{REFERENCE NOTE}

1. McGee, R., \& Golus, P. Effects of two different types of anesthetics on neophobia in the rat: Sodium pentobarbital and ketamine $\mathrm{HCl}$. Paper presented at the Australian Neurosciences Society Conference, Canberra, Australia, 1980.

\section{REFERENCES}

Broadhurst, P. L. Experiments in psychogenetics: Application of biometrical genetics to the inheritance of behavior. In H. J. Eysenck (Ed.), Experiments in personality (Vol. 1). London: Routledge \& Kegan Paul, 1960.

Cunha, J. M., \& MAsur, J. Evaluation of psychotropic drugs with a modified open field test. Pharmacology, 1978, 16, 259-267.

HARRIS, R. A., \& CASE, J. Effects of maternal consumption of ethanol, barbital or chlordiazepoxide on the behavior of the of fspring. Behavioral and Neural Biology, 1979, 26, 234-237.

HenNig, C. W. Biphasic effects of serotonin on tonic immobility in domestic fowl. Pharmacology, Biochemistry and Behavior, 1979, 12, 519-523.

JACOBS, B. L., \& FAREL, P. B. Motivated behaviors produced by increased arousal in the presence of goal objects. Physiology \& Behavior, 1971, 6, 473-476.

Kuschinsky, K., Seidel, G., Reetz, E., \& Meyer-Burgdorf, C. The effect of pentobarbital on brain 5-HT metabolism in mice. Experientia, 1973, 29, 826-827.

Mattingley, D. A. A simple fluorometric method for estimation of free 11-hydroxycorticosteroids in human plasma. Journal of Clinical Pathology, 1962, 15, 374-379.

McGee, R., Golus, P., \& Russell-Jones, C. Long-term effect of pentobarbital anesthesia on neophobia in the rat. Behavioral and Neural Biology, 1981, 32, 376-379.

Pfister, H. P., \& King, M. G. Adaptation of the glucocorticosterone response to novelty. Physiology \& Behavior, 1976, 17, 43-46.

Power, P., Myers, W., Golus, P., \& McGee, R. Long-term effects of barbiturate anesthesia on whole brain monamine levels in the rat. IRCS Medical Science, 1980, 8, 630.

Rondeau, D. B., Jolicoeur, F. B., Kachanoff, R., Scherzer, P., \& WAYNER, M. J. Effects of phenobarbital on ethanol intake in fluid deprived rats. Pharmacology, Biochemistry and Behavior, 1975, 3, 493-497.

Watson, P. J., \& Cox, V. C. An analysis of barbiturate-induced eating and drinking in the rat. Physiological Psychology, 1976, 4, 325-332.

(Manuscript received November 1, 1980; revision accepted for publication August 4, 1981.) 